\title{
A Case of Abdominal Cocoon Syndrome: A Rare Cause of Small Bowel Obstruction At Aber Hospital in Northern Uganda
}

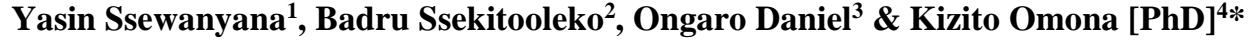 \\ ${ }^{1}$ Surgeon, Lira Regional Referral Hospital, Lira District (Uganda) \\ ${ }^{2}$ General Surgeons, Mulago National Referral Hospital \& Makerere College of Health Sciences (Uganda) \\ ${ }^{3}$ Medical Officer Intern, Aber Hospital-Oyam District (Uganda) \\ ${ }^{4}$ Lecturer, Uganda Martyrs University, Faculty of Health Sciences, P. O. Box 5498, Kampala, Uganda \\ *Corresponding author E-mail:komona@umu.ac.ug
}

\begin{abstract}
Abdominal cocoon syndrome (ACS) is a rare cause of small bowel obstruction characterized by partial or complete encasement of small bowel loops in a thick fibro-collagenous sac. It poses diagnostic difficulties due to the non-specific nature of its presentation and thus in most cases, diagnosed incidentally at laparotomy.

The researchers present a case of a 27 -year old female Ugandan who presented to a rural hospital in Northern Uganda with complaints of central abdominal pain, failure to pass stool and bilious vomiting for three days. She also had feelings of a peri-umbilical mass. She had several episodes of related obstructive symptoms that would be self-limiting for a year. She had unremarkable past surgical, past medical and past gynecological history. Laboratory investigations were unremarkable.

Preoperative diagnosis of small bowel intussusception was made basing on examination and ultrasonography report and the patient was managed operatively. At laparotomy, almost all the small bowel loops were found encased in a thick, whitish, fibrous membrane. There was also marked inter-loop adhesions. The membranous sac was incised and completely removed using both sharp and blunt dissection. Inter-loop adhesions were released solely by blunt dissection.

The patient recovered postoperatively and was discharged on the seventh postoperative day.
\end{abstract}

Keywords: Abdominal Cocoon Syndrome; Small Bowel Obstruction; Laparotomy; Intussusceptions.

\section{Introduction}

Abdominal cocoon syndrome (ACS), also known as Sclerosing Encapsulating Peritonitis (SEP), is a rare cause of small bowel obstruction that is often never suspected and thus diagnosed incidentally. It is characterized by partial or complete encasement of small bowel by a thick, white fibro-collagenous membrane (Gupta, et al., 2013; Sharma, et al., 2013; Yavuz, et al., 2015). This condition was first described in 1907 by Owtschinnikov who termed it "Peritonitis Chronica Fibrosa Incapsulata" while Foo (1978) coined the term "Abdominal cocoon syndrome" in 1978(Tannoury and Abboud 2012). The researchers present a case that was diagnosed incidentally at laparotomy in a rural Hospital in Northern Uganda. To the best of our knowledge, there are no published cases of this condition in Uganda.

\section{Case Presentation}

\subsection{Patient demographics}

She was a 27 year old female, a Luo from Ogwala-Agoa village in Oyam district of Northern Uganda, peasant farmer, Born Again Christian and married. She attained primary level education. She was admitted on 25/04/2019, operated on $26^{\text {th }}$ April 2019 and discharged on $3^{\text {rd }}$ May 2019.

\subsection{Presenting complaint}

The patient presented with abdominal pain, failure to pass stool for 3 days and vomiting for 2 days.

\subsection{History of presenting complaint}


Patient was relatively well till 3 days prior to admission when she developed a gradual onset of peri-umbilical and right upper quadrant colicky abdominal pain. This was associated with moderate peri-umbilical distension more to the right and feeling of a mass in the same area. She was unable to pass stool (but occasionally passing flatus), had non-projectile vomiting that was initially non-bilious but later progressively became bilious. The pain was aggravated on food intake, mildly relieved on vomiting.

She had suffered several episodes of related symptoms over a period of a year that could get relieved after a few days without medical intervention and with complete disappearance of the associated mass.

No history of fevers related to the above symptoms was reported. She had been having normal appetite and denied any feelings of weight loss.

She had unremarkable past surgical history with no imaging ever done to investigate the cause of her problem.

\subsection{Past medical history}

She was HIV sero-negative (tested at admission) and not known for any chronic medical illnesses and never been on prolonged medication.

\subsection{Review of other systems}

Review of other systems was unremarkable

\subsection{Gynaecological history}

She had menarche at 15 years. She reported normal, painless menses that lasts 5 to 6 days, and a menstrual cycle of approximately 30 days. She reported episodes of pelvic pain prior to conception, ten years ago, that were not associated with Per Vaginal (PV) discharge and menstruation with symptoms improving on unspecified medication. No reported history of an abortion or ectopic pregnancy or any gynecological procedures performed on her.

She had one (1) sexual partner in life. No history of dyspareunia or post coital bleeding. She used Injectaplan (DMPA) for one year (2014) for contraception. At the time of admission she was not on any contraceptive method.

\subsection{Family-social history}

She is married and has two children, who are alive and well. She stopped taking alcohol (traditional local brew) one year ago when she started having symptoms of this current illness. She does not smoke cigarette.

\subsection{Summary}

A 27 year old female who came to Aber Hospital with history of colicky abdominal pain, failure to pass stool but occasionally passing flatus, non-projectile bilious vomiting, moderate peri-umbilical abdominal distension. She had had several episodes of self-limiting related symptoms over a year. She had unremarkable past surgical, past medical and past gynaecological history.

\subsection{General examination}

A young adult female in fair nutritional status, not obviously in pain, not in respiratory distress, afebrile (axillary temperature, $37.5^{\circ} \mathrm{C}$ ) She was not jaundiced, had no pallor, no cyanosis, no digital clubbing, no palpable lymphadenopathy, or pedal edema.

Vitals findings; Blood pressure was $\frac{100}{60} \mathrm{mmHg}$, Pulse Rate (PR) was 85beats per minute (good volume and regular), Respiratory Rate (RR) was 17 breath per minute and $\mathrm{SPO}_{2}$ was $99 \%$ on room air.

\subsection{Abdominal examination}

The abdomen was of normal fullness and moving with respiration. No visible collateral vessels or therapeutic and surgical scars. Umbilicus was normally inverted, no evidence of abdominal wall defects. There was a visible central mass more marked in the right upper quadrant. It was about $10.5 \mathrm{~cm}$ in greatest dimension, mildly tender, ovoid, firm, smooth, slightly mobile, and non-pulsatile. The mass had a dull percussion note while the remaining parts of the abdomen had normal resonance. Bowel sounds appeared reduced.

\subsection{Digital rectal examination}

There was normal peri-anal area, with normal anal sphincter tone, rectal wall was smooth with no palpable mass(es). There was hard formed stool.

\subsection{Other systemic examinations}

Findings on other system examination were unremarkable

\subsection{Findings of investigations}

- HIV serology (Negative), Blood group (O Rhesus Positive)

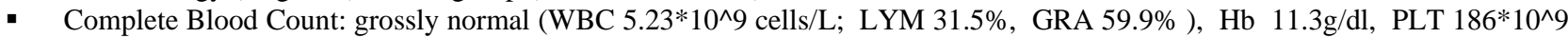
cells/L)

- Serum Electrolytes: normal $(\mathrm{K}+3.48 \mathrm{mmol} / \mathrm{L}, \mathrm{Na}+136.7 \mathrm{mmom} / \mathrm{L}, \mathrm{Cl}-99.3 \mathrm{mmol} / \mathrm{L})$ 
- Abdominal ultrasound scan; There is a poorly defined tender "target like" concentric rings of bowel loops seen with a bright echogenic centre on TS and with multiple layers on LS seen in the RLQ. The mass shows no peristalsis and is surrounded by a thin rim of free fluid. Impression was small bowel intussusception.

- Plain abdominal X-ray showed equivocal findings

\subsection{Treatment}

Pre-operative treatment: It included but not limited to: NPO, NGT decompression, and IV Fluids, analgesia and antibiotics administration.

Intra-operative findings: A massive peritoneal adhesions onto the anterior abdominal wall was found and released. No visible bowel loops on opening. A sac-like fibrous membranous structure was enclosing almost all the small bowel loops. Adhesiolysis was done and gentle separation of sac-like structure from the bowel loops using combination of blunt and sharp dissection was done. An ileal perforation of about $30 \mathrm{~cm}$ from the ileocecal junction was got and repaired transversely. Appendectomy and peritoneal lavage of the abdomen with $3 \mathrm{~L}$ of warm saline was done and the abdomen was closed in respective layers.

Postoperative Management: The post-operative treatment included but not limited to the following;

- NPO, maintained NGT for 72 hours

- Continuous bladder drainage for 48 hours

- IV Fluids

- IV Antacids (Ranitidine)

- Analgesia

- IV antibiotics (Ceftriaxone and metronidazole).

Postoperative follow up: The patient had an uneventful recovery. She was started on oral sips on postoperative day 3 and started soft feeds on postoperative day 4. She was allowed liberal diet on postoperative day 5 and discharged on postoperative day 7 with marked improvement. Figure 1, 2, \& 3 below show some of the clinical findings. Figure 1 was pre-operative finding whereas figure $2 \& 3$ are intra-operative findings.

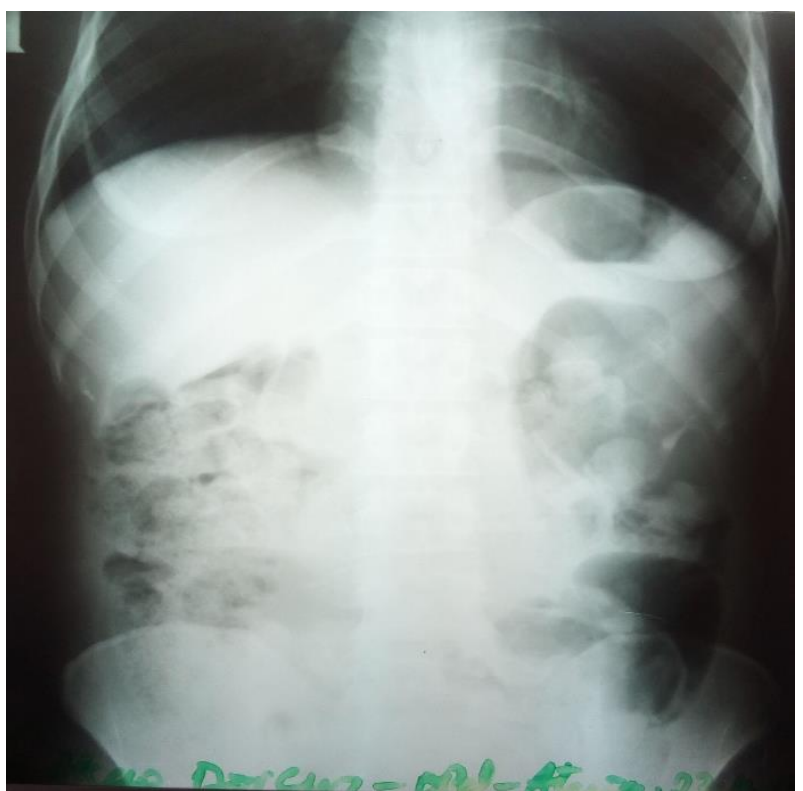

Fig.1:The Patient's Plain Erect Abdominal Radiograph 


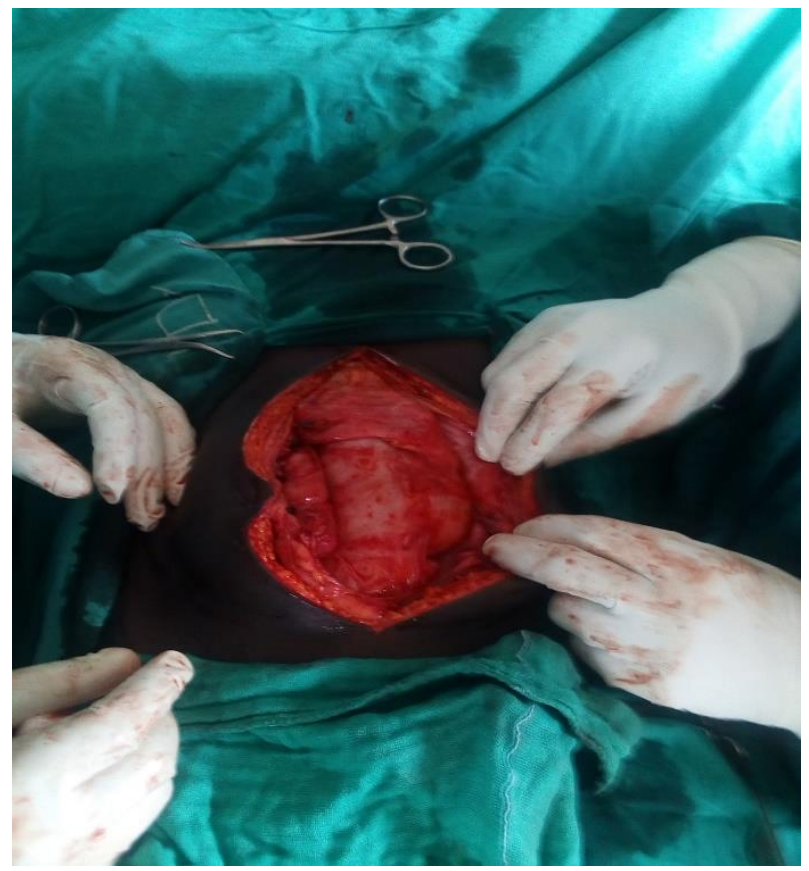

Fig. 2: Appearance of the Peritoneal Cavity Upon Opening, No Bowel Loops Visible

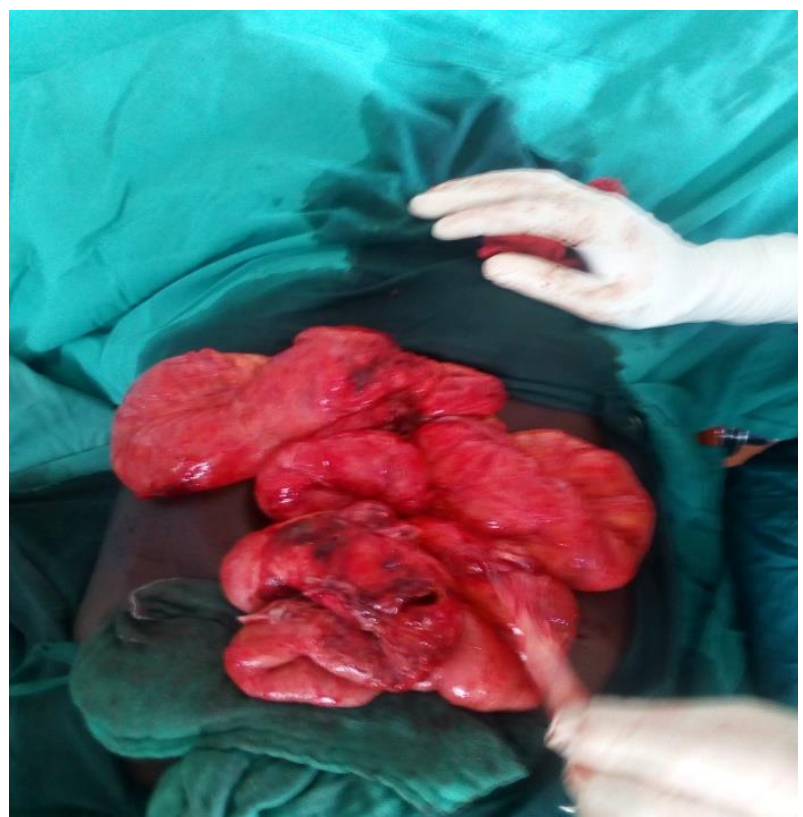

Fig. 3:Small Bowel Loops After Removal of the Encapsulating Membrane and Inter-Loop Adhesiolysis. Ileal Perforation Sustained Was Repaired Transversely

\section{Discussion}

Although it was thought to be more common among female adolescents in tropical and subtropical countries (Solak and Solak, 2012; Yavuz, Akbulut, et al., 2015), ACS affects both males and females across a range of age groups (Deeb, Mourad, et al., 1998; Matone, Herbella, et al., 2006). Cases have also been reported in temperate zones of the world. The oldest documented case was a ninety year old male from Turkey in 2011(Yavuz, Akbulut, et al., 2015). This patient (27year old) was a female from the tropics but well above the adolescent age bracket. It is possible, however, that she developed the disease during adolescence but only presented later.

The etiology of abdominal cocoon syndrome has remained unclear up to date. Abdominal cocoon syndrome can be classified as primary (idiopathic) or secondary depending on whether or not a definite cause can be identified. In females, hypotheses suggest possible etiologies for idiopathic ACS to include retrograde menstruation and retrograde extension of fallopian tube infections or viral gynecological infection (Sieck, Cowgill, et al., 1983; Yavuz, Akbulut, et al., 2015). In males, it is also thought that there is an infectious cause (Ibrahim and Oludara, 2009). Some of the suggested predisposing factors for secondary ACS include; previous surgeries, sarcoidosis, Systemic Lupus Erythematosus (SLE), gastrointestinal tumors, ventriculoperitoneal and peritoneovenous shunts, fibrogenic foreign body, recurrent peritonitis, abdominal tuberculosis and prolonged use of beta adrenergic blockers among others (Nakamoto, 2005; Matone, Herbella, et al., 2006; Suh, Lee, et al., 2007, Taylor, Clarke, et al., 2012; Singh and Gupta, 2013; Yavuz, Akbulut, et al., 2015). In this patient, the investigating team was not able to elicit any risk factors to suggest a secondary form of ACS and so we think it was a case of idiopathic ACS. Majority of previously reported cases were also thought to be idiopathic.

Some cases of this condition may remain asymptomatic for several years. Majority, however, are characterized by recurrent bouts of acute, sub-acute or chronic intestinal obstruction that get relieved without specific treatment (Xu, Chen, et al., 2007; Tannoury and 
Abboud, 2012; Taylor, Clarke, et al., 2012). These symptoms are secondary to kinking or constriction of the intestines within the constricting membranous cocoon. Some patients also have an abdominal mass which is basically due to an encapsulated cluster of small bowel loops (Hur, Kim, et al., 2004; Gupta, Gupta, et al., 2013; Sharma, Nair, et al., 2013). This patient started having obstructive symptoms a year prior to this admission and reported complete relief of symptoms in between episodes without specific treatment. This presentation is not so different from those of several other cases reported. It is possible that she had an asymptomatic course of the disease long before symptoms appeared just a year prior to the diagnosing laparotomy.

Preoperative diagnosis of ACS is still highly challenging even to the most experienced practitioners due to the non-specific nature of its presentation. Only imaging can attempt to provide a preoperative diagnosis and modalities used include; abdominal ultrasonography, plain erect abdominal radiographs, barium studies (particularly barium follow-through), and computed tomography (CT). Many of these may still give unspecific results. Abdominal ultrasonography may demonstrate dilated small bowel segments surrounded by a thick, hypoechoic fibrous membrane (Gupta, Gupta, et al., 2013). This, however, depends on the expertise of the sonographer but it may also be missed. For this case, the sonographer reported features suggestive of small bowel intussusceptions and it was upon this report that an emergency laparotomy was preferred.

Plain erect abdominal radiograph is unspecific and usually shows dilated small bowel loops with multiple air-fluid levels, only suggestive of small bowel obstruction with no attempt to suggest a cause. The plain radiograph of this patient was even more confusing, as it did not have the common features of obstruction.

If done, a barium follow-through may show a serpentine configuration of the dilated distal bowel within the cocoon(Sieck, Cowgill, et al., 1983). Some literature reports that findings of an accordion pattern and cauliflower appearance may give a clue to aid preoperative diagnosis (Navani, Shah, et al., 1995; Xu, Chen, et al., 2007; Yavuz, Akbulut, et al., 2015). This was not done for this patient.

An abdominal CT scan is currently the most useful radiological investigation, especially the Multidetetor CT with excellent images on coronal, sagittal andaxial planes. The characteristic sign is the appearance of loops of small bowel that conglomerate at midline and are encased by a dense mantle without peripheral uptake of the contrast (Wig and Gupta, 1998; Serter, Kocakoç, et al., 2013; Vagholkar and Doctor-Ganju, 2018). This patient could not access this test as it would mean travelling a distance of more than 300killometeres to Uganda's capital of Kampala. It is also not common practice in Uganda to do abdominal CTs for all patients with features intestinal obstruction due to limitations of both physical and financial access to the service. Moreover, the clinical picture combined with the differential of intussusception suggested by ultrasonography had already biased the decision of the team to undertake an exploratory laparotomy.

The final diagnosis of ACS is usually made basing on intra-operative findings and histopathology results. The intra-operative findings are of small bowel loops being partially or completely encased in a fibrous, sac-like structure. For this patient, there was no clue to this diagnosis preoperatively. The entire small bowel, from the ligament of Treitz to a few centimetres from the ileocecal junction was encased in a thick, whitish, fibrous sac.

Intra-operative treatment consists of excising the accessory peritoneal sac and lysis of the inter-loop adhesions and this is what was done for the present case. As it was for this case, bowel resection is usually not necessitated unless a non-viable segment is found. Many scholars recommend incidental appendectomy to be performed on these patients (Taylor, Clarke, et al., 2012). In this case, the surgeon performed appendectomy just to save them the burden of future appendicitis.

As it was for this present case, patients usually have a good postoperative prognosis with complete remission of symptoms. Our patient had an uneventful postoperative period and was discharged on the seventh postoperative day.

\section{Conclusion}

Abdominal cocoon syndrome remains a mystery to the surgeons with poorly understood etiology, pathogenesis and difficulties in obtaining a preoperative diagnosis. Despite advances in radiological imaging, the condition is still mostly diagnosed incidentally at laparotomy. In the developing world, the diagnosis is likely to remain mainly incidental (at laparotomy) due to the limited access to the imaging modalities that would attempt a preoperative diagnosis. Recognition of the condition is important for better management and prevents unnecessary bowel resection that would otherwise increase morbidity and mortality.

\section{Acknowledgement}

The authors do acknowledge all the support and corporation of staff of Aber Hospital. Most importantly, special appreciation goes to our client without whom this case report would not be possible.

\section{Funding}

No external funding was provided for this study.

\section{Competing interest}

The authors declare no competing interest of any form.

\section{References}

[1] Deeb, L. S., F. H. Mourad, Y. R. El-Zein and S. M. Uthman (1998). "Abdominal cocoon in a man: preoperative diagnosis and literature review." Journal of clinical gastroenterology 26(2): 148-150.https://doi.org/10.1097/00004836-199803000-00013.

[2] Gupta, S., A. Gupta, C. Yadav and A. Dwivedi (2013). "Abdominal cocoon: Case report and literature review." Sch J App Med Sci 1: 748-752.

[3] Hur, J., K. W. Kim, M.-S. Park and J.-S. Yu (2004). "Abdominal cocoon: preoperative diagnostic clues from radiologic imaging with pathologic correlation." American Journal of Roentgenology 182(3): 639-641.https://doi.org/10.2214/air.182.3.1820639.

[4] Ibrahim, N. and M. Oludara (2009). "Abdominal cocoon in an adolescent male patient." Tropical doctor 39(4): 254256.https://doi.org/10.1258/td.2009.090104. 
[5] Matone, J., F. Herbella and J. C. Del Grande (2006). "Abdominal cocoon syndrome." Clinical Gastroenterology and Hepatology 4(5)https://doi.org/10.1016/j.cgh.2005.12.005.

[6] Nakamoto, H. (2005). "Encapsulating peritoneal sclerosis--a clinician's approach to diagnosis and medical treatment." Peritoneal Dialysis International 25(Suppl 4): S30-S38.https://doi.org/10.1177/089686080502504S05.

[7] Navani, S., P. Shah, S. Pandya and N. Doctor (1995). "Abdominal cocoon--the cauliflower sign on barium small bowel series." Indian journal of gastroenterology 14(1): 19-19.

[8] Serter, A., E. Kocakoç and G. Çipe (2013). "Supposed to be rare cause of intestinal obstruction; abdominal cocoon: report of two cases." Clinical Imaging 37(3): 586-589.https://doi.org/10.1016/j.clinimag.2012.08.010.

[9] Sharma, D., R. P. Nair, T. Dani and P. Shetty (2013). "Abdominal cocoon-A rare cause of intestinal obstruction." International journal of surgery case reports 4(11): 955-957.https://doi.org/10.1016/j.ijscr.2013.08.004

[10] Sieck, J. O., R. Cowgill and W. Larkworthy (1983). "Peritoneal encapsulation and abdominal cocoon: case reports and a review of the literature." Gastroenterology 84(6): 1597-1601.https://doi.org/10.1016/0016-5085(83)90387-6.

[11] Singh, B. and S. Gupta (2013). "Abdominal cocoon: a case series." International Journal of Surgery 11(4): 325328.https://doi.org/10.1016/j.ijsu.2013.02.011.

[12] Solak, A. and İ. Solak (2012). "Abdominal cocoon syndrome: preoperative diagnostic criteria, good linical outcome with medical treatment and review of the literature." The Turkish journal of gastroenterology 23(6): 776-779.https://doi.org/10.4318/tjg.2012.0500.

[13] Suh, W. N., S. K. Lee, H. Chang, H. J. Hwang, W. J. Hyung, Y. N. Park and T. I. Kim (2007). "Sclerosing encapsulating peritonitis (abdominal cocoon) after abdominal hysterectomy." The Korean journal of internal medicine 22(2): 125.https://doi.org/10.3904/kjim.2007.22.2.125.

[14] Tannoury, J. N. and B. N. Abboud (2012). "Idiopathic sclerosing encapsulating peritonitis: abdominal cocoon." World journal of gastroenterology 18(17): 1999.https://doi.org/10.3748/wjg.v18.i17.1999.

[15] Taylor, M., M. G. Clarke, J. Jarvis and M. Booth (2012). "A mystery wrapped in an enigma: The abdominal cocoon syndrome." NZ Med J125: 7780.

[16] Vagholkar, K. and K. Doctor-Ganju (2018). "Abdominal cocoon: a surgical challenge." International Surgery Journal 5(12): 40904094.https://doi.org/10.18203/2349-2902.isj20184713.

[17] Wig, J. and S. Gupta (1998). "Computed tomography in abdominal cocoon." Journal of clinical gastroenterology 27(3): 259260.https://doi.org/10.1097/00004836-199810000-00018.

[18] Xu, P., L.-H. Chen and Y.-M. Li (2007). "Idiopathic sclerosing encapsulating peritonitis (or abdominal cocoon): a report of 5 cases." World journal gastroenterology 13(26): 3649.https://doi.org/10.3748/wjg.v13.i26.3649.

[19] Yavuz, R., S. Akbulut, M. Babur and F. Demircan (2015). "Intestinal obstruction due to idiopathic sclerosing encapsulating peritonitis: a case report." Iranian Red Crescent Medical Journal 17(5).https://doi.org/10.5812/ircmj.17(5)2015.21934. 